\title{
FLORÍSTICA E ESTRUTURA DO COMPONENTE ARBÓREO DE UMA FLORESTA OMBRÓFILA DENSA MONTANA EM SANTA CATARINA, BRASIL ${ }^{1}$
}

\author{
Dolores Martins Bosa², Dilton Pacheco², Marcelo Romagna Pasetto ${ }^{2}$ e Robson Santos ${ }^{3}$ \\ RESUMO - Para ampliar o conhecimento sobre a Floresta Ombrófila Densa Montana no Estado de Santa \\ Catarina, foi realizado estudo florístico e fitossociológico do componente arbóreo. Neste estudo, foi usado \\ o método do ponto quadrante (250 pontos), incluindo todos os indivíduos com Diâmetro à Altura do Peito \\ (DAP) mínimo de $5 \mathrm{~cm}$. A composição florística resultou em riqueza total de 102 espécies. A densidade \\ total arbórea foi de 2.034 indivíduos.ha ${ }^{-1}$. As famílias com maior número de espécies foram Myrtaceae, \\ Lauraceae, Fabaceae e Annonaceae, e entre as espécies com maior valor de importância estão Euterpe edulis \\ Mart. e Ficus adathodifolia Schott ex Spreng. A classificação das espécies arbóreas em grupos ecológicos \\ indicou ligeira predominância de espécies dos estádios finais (56\%) sobre as espécies dos estádios iniciais \\ da sucessão, indicando que a vegetação se encontra em estádio avançado da sucessão ecológica. A síndrome \\ de dispersão predominante entre as espécies arbóreas foi a zoocoria, identificada em $77 \%$ delas, seguida \\ pela anemocoria, que representou $15 \%$ dos casos. Os resultados apresentados contribuirão para posteriores \\ estudos de dinâmica em remanescentes de Floresta Ombrófila Densa Montana e para programas de manejo, \\ preservação e recuperação ambiental.
}

Palavras-chave: Sucessão ecológica; Remanescentes florestais; Grupos sucessionais.

\section{FLORISTIC AND STRUCTURE OF THE TREE COMPONENT OF AN ATLANTIC RAINFOREST IN SANTA CATARINA STATE, BRAZIL}

\begin{abstract}
To enlarge the knowledge of an Atlantic rainforest in Santa Catarina State, a floristic and phytosociological study of the tree component was carried out. In this study, the quadrant-point method (250 points) was used, including all the individuals with minimum DBH (diameter at breast height) of $5 \mathrm{~cm}$. The floristic composition resulted in a total richness of 102 species. The tree total density was 2,034 individuals. ha ${ }^{-1}$. The families with the highest number of species were Myrtaceae, Lauraceae, Fabaceae, and Annonaceae, and among species with highest importance value are Euterpe edulis Mart. and Ficus adathodifolia Schott ex Spreng. The tree species classification in ecological groups showed a slight predominance of the late secondary species (56\%) over species of early succession, indicating that the vegetation is in the advanced stage of ecological succession. Zoochory was the most frequently observed syndrome (77\%), followed by anemochory (15\%). The results will contribute to further studies on remnant of Atlantic Rainforest in Santa Catarina State dynamic and to environmental management, preservation and recovery programs.
\end{abstract}

Keywords: Ecological succession; Forest remnant; Successional groups.

\footnotetext{
${ }^{1}$ Recebido em 08.05.2013 aceito para publicação em 19.11.2014.

${ }^{2}$ Universidade do Extremo Sul Catarinense (UNESC), Mestrado em Ciências Ambientais, Criciúma, SC - Brasil. E-mail: <doloresmbosa@yahoo.com.br>,<diltontur@hotmail.com>e<marcelo.pasetto@hotmail.com>.

${ }^{3}$ Universidade do Extremo Sul Catarinense (UNESC), Programa de Pós-Graduação em Ciências Ambientais, Herbário Padre Dr. Raulino Reitz (CRI), Criciúma, SC - Brasil. E-mail: <rsa@unesc.net>.
} 


\section{INTRODUÇÃO}

O Brasil é o país de maior biodiversidade do planeta, com um patrimônio natural reconhecido como o mais significativo do mundo (BRITTO, 2006). Sua maior riqueza é expressa pela diversidade e endemismo das espécies biológicas e pelo seu patrimônio genético, bem como pela variedade ecossistêmica dos biomas (ARRUDA, 2001).

A conservação da biodiversidade regional é sem dúvida um dos maiores obstáculos a serem superados neste século, isso em razão do elevado nível de perturbações pela ação humana, aos quais estão expostos os ecossistemas naturais. Atualmente, o bioma Mata Atlântica encontra-se reduzido a fragmentos florestais isolados $(<100 \mathrm{ha})$, indicando que restam aproximadamente $11 \%$ da vegetação original (RIBEIRO et al., 2009). Apesar disso, estima-se que esse bioma abriga 20.000 espécies de plantas vasculares, das quais cerca de 8.000 são endêmicas (MYERS et al., 2000).

Na região Sul do Estado de Santa Catarina, apesar dos impactos da poluição da mineração de carvão e da degradação resultantes da extração e deposição de rejeitos e estéreis da mineração, a atividade agrícola foi o principal fator que desencadeou a fragmentação da floresta original (NASCIMENTO, 2003). As florestas mais preservadas encontram-se nas encostas da Serra Geral.

As encostas da Serra Geral, por sua grande altitude e posição próxima ao oceano, exercem grande influência na distribuição regional das chuvas, agindo como barreira que provoca sua precipitação pela condensação das nuvens provenientes do oceano. Com sua vegetação original em grande parte preservada, essas áreas são essenciais para a regulação de toda a disponibilidade hídrica das bacias dos rios Araranguá e Mampituba. Nessa região, a Floresta Ombrófila Densa Montana desempenha papel de grande relevância no controle dos processos erosivos, bem como na infiltração e liberação gradativa da água das chuvas, sendo, portanto, responsável pela manutenção das nascentes dos principais rios das bacias hidrográficas da região. Considerando a grande declividade das encostas, sua manutenção está protegida pelo código florestal, porém se encontra permanentemente ameaçada pela indústria madeireira, pelas enxurradas e pelo fogo (SCHEIBE et al., 2010).

Revista Árvore, Viçosa-MG, v.39, n.1, p.49-58, 2015
Discutir preservação torna-se, dessa forma, tarefa complexa, uma vez que é necessário explorar os recursos naturais para continuar usufruindo de seus benefícios. Isso, porém, envolve interesses, perspectivas e limitações, seja quanto ao método, seja quanto ao domínio do conhecimento das relações entre os elementos da natureza. Conhecer a diversidade biológica nos remanescentes florestais, por meio de sua quantificação, e a compreensão da organização espacial das comunidades fornece conhecimento sobre parte da riqueza florestal do país ainda não conhecida.

Nesse contexto, buscou-se compreender a composição florística e estrutural da comunidade arbórea de um remanescente de Floresta Ombrófila Densa Montana na localidade de Três Barras, Município de Morro Grande, Sul do Estado de Santa Catarina.

\section{MATERIAL E MÉTODOS}

\subsection{Localização da área}

O estudo foi realizado no Município de Morro Grande (28 44' S, 49 $45^{\prime} \mathrm{W}$ ), situado no Sul de Santa Catarina, na Bacia Hidrográfica do Rio Araranguá. O clima da região, segundo a classificação de Köppen, é do tipo Cfa e $\mathrm{Cfb}$, ou seja, clima subtropical úmido sem estação seca definida, com verão quente (Cfa) e com verão ameno $(\mathrm{Cfb})$ nas regiões mais altas. A temperatura média anual do município varia de 17 a $19^{\circ} \mathrm{C}$, e a precipitação média anual é de $1.600 \mathrm{~mm}$, com o total anual de dias de chuva entre 102 e 150 dias (PANDOLFO et al., 2002).

O núcleo colonial que deu origem ao Município de Morro Grande surgiu no início do século XX, com a chegada das primeiras famílias, descendentes de italianos provindos dos Municípios de Criciúma, Içara, Morro da Fumaça e Urussanga, em Santa Catarina. Além da derrubada da floresta para a agricultura e criação de animais, a prática social dos agricultores foi aumentando o processo de fragmentação florestal. A base da economia é a agricultura, com destaque para o cultivo de arroz, milho, feijão, banana e beneficiamento de madeira.

Segundo relato de morador local, parte da área estudada foi utilizada para a agricultura (45 anos atrás). Nessa área, cultivaram-se, principalmente, milho e feijão, que serviam de alimento para as famílias que viviam na localidade. O difícil acesso a essas áreas e as constantes enchentes que destruíam as vias de acesso 
mantiveram, muitas vezes, os moradores isolados, o que provocou o seu deslocamento para outras localidades, abandonando a agricultura da área para trabalharem em outras com acesso facilitado. Há 30 anos, a área foi utilizada para extração seletiva de madeira e logo depois abandonada, não sendo mais utilizada para nenhuma atividade.

\subsection{Metodologia}

O levantamento fitossociológico foi realizado com o uso do método de ponto quadrante (COTTAM; CURTIS, 1956), sendo amostrados todos os indivíduos arbóreos (incluindo palmeiras e samambaias arborescentes) com diâmetro do caule à altura do peito (DAP) mínimo de $5 \mathrm{~cm}$. Foram alocados 250 pontos amostrais (amostragem total de 1.000 árvores). Foi obedecido um intervalo de $10 \mathrm{~m}$ entre pontos em linhas paralelas, distantes entre si $50 \mathrm{~m}$. Com base nos dados de campo, foram calculados os seguintes parâmetros fitossociológicos para as espécies: frequência, densidade e dominância absolutas e valor de importância, de acordo com MuellerDombois e Ellenberg (1974).

O material coletado fértil foi depositado no Herbário Padre Dr. Raulino Reitz (CRI), da Universidade do Extremo Sul Catarinense (UNESC). Foi adotado o sistema APG III (APG, 2009) para classificação das famílias botânicas. A atualização taxonômica das espécies e de seus autores seguiu a Lista de Espécies da Flora do Brasil (2014).

Para cada espécie arbórea foram fornecidas informações sobre grupo ecológico (pioneira, secundária inicial, secundária tardia e clímax) e síndromes de polinização e de dispersão. Para os grupos ecológicos, seguiu-se a proposta de Ferretti et al. (1995) e para as síndromes de polinização e de dispersão, os conceitos estabelecidos de Faegri e van der Pijl (1979) e van der Pijl (1982), respectivamente.

\section{RESULTADOS}

Foram identificadas 102 espécies arbóreas, distribuídas em 78 gêneros e 42 famílias, sendo, entre essas, duas espécies exóticas invasoras. Foram amostrados 1.000 indivíduos, com densidade total de 2.034 indivíduos.ha-1 ${ }^{-1}$ Tabela 1). Das famílias registradas, $21(21 \%)$ estavam representadas por apenas uma espécie, seis $(6 \%)$ por duas espécies e sete $(7 \%)$ por três. Myrtaceae apresentou a maior riqueza específica, com 11 espécies (11\%), sendo cinco pertencentes ao gênero
Myrcia. Após, seguiram-se Lauraceae, com sete espécies (7\%), Fabaceae e Annonaceae, com seis cada (6\%). Essas quatro famílias detiveram $30 \%$ das espécies amostradas.

Com relação à densidade e frequência, Euterpe edulis Mart. destacou-se pelo maior número de indivíduos (645 indivíduos.ha ${ }^{-1}$ ) e apresentou maior ocorrência, estando presente em 72 das 250 unidades amostrais. O valor obtido para área basal total foi de $59 \mathrm{~m}^{2} \cdot \mathrm{ha}^{-1}$, sendo a espécie com maior dominância e contribuição nesse parâmetro Ficus adathodifolia Schott ex Spreng., com $12 \mathrm{~m}^{2} \cdot \mathrm{ha}^{-1}$.

Os valores de diâmetro (DAP) variaram de $5 \mathrm{~cm}$ até $95 \mathrm{~cm}$. Aproximadamente, $75 \%$ do total concentrou-se entre 5,0 e $14,9 \mathrm{~cm} ; 14 \%$ entre 15,0 e $24,9 \mathrm{~cm}$; e apenas $11 \%$ apresentou caules iguais ou superiores a $55,0 \mathrm{~cm}$ (Figura 1). O maior valor diamétrico foi obtido por $F$. adathodifolia $(95 \mathrm{~cm})$, seguido por indivíduos de Hyeronima alchorneoides Allemão e Erythrina falcata Benth., com $63 \mathrm{~cm}$ e $53 \mathrm{~cm}$ de diâmetro, respectivamente.

As espécies de maior valor de importância (VI) foram E. edulis, F. adathodifolia, Nectandra membranacea (Sw.) Griseb., Bathysa australis (A.St.Hil.) K. Schum., H. alchorneoides, Psychotria suterella Müll. Arg., Alchornea triplinervia (Spreng.) Müll. Arg., Cabralea canjerana (Vell.) Mart., Alsophila setosa Kaulf. e Matayba guianensis Aubl. Essas 10 espécies totalizaram $61 \%$ dos indivíduos amostrados.

A altura dos indivíduos registrados neste estudo variou de 2 a $20 \mathrm{~m}$, com a altura média de 6,5 m (Figura 2). As espécies encontradas no estrato inferior da área de estudo foram $A$. setosa, $P$. suterella, Rudgea jasminoides (Cham.) Müll. Arg. e Mollinedia schottiana (Spreng.) Perkins. Aproximadamente, 56\% dos indivíduos amostrados ficaram concentrados no estrato inferior. No estrato médio, a maior representatividade deu-se pelos indivíduos de E. edulis, B. australis, C. canjerana, Trichilia lepidota Mart. e Gymnanthes concolor Spreng. Entre as espécies que se concentraram no estrato superior, destacaram-se F. adathodifolia, H. alchorneoides e Cinnamomum glaziovii Mez, todas com $20 \mathrm{~m}$.

Analisando a comunidade arbórea em relação aos grupos ecológicos das 100 espécies nativas encontradas, obtiveram-se $20 \%$ de pioneiras, $24 \%$ de secundárias iniciais, $37 \%$ de secundárias tardias e 19\% de clímax. Com relação às síndromes de polinização e de dispersão, 
Tabela 1 - Parâmetros estruturais das espécies arbóreas de uma Floresta Ombrófila Densa Montana, no Município de Morro Grande, Santa Catarina, para indivíduos com DAP $\geq 5 \mathrm{~cm}$. Em que $\mathrm{H}=$ altura máxima $(\mathrm{m}) ; \mathrm{FA}=$ frequência absoluta (\%); DoA = dominância absoluta $\left(\mathrm{m}^{2} . \mathrm{ha}^{-1}\right) ; \mathrm{DA}=$ densidade absoluta (indivíduos.ha $\left.{ }^{-1}\right)$; VI = valor de importância; e Ec = grupo ecológico, sendo $\mathrm{P}=$ pioneira, $\mathrm{Si}=$ secundária inicial, $\mathrm{St}=$ secundária tardia, $\mathrm{C}=$ clímax, $\mathrm{P}=$ síndrome de polinização, em que $\mathrm{A}$ = anemofilia, $\mathrm{Z}$ = zoofilia e $\mathrm{D}=$ síndrome de dispersão, sendo $\mathrm{An}=$ anemocoria, $\mathrm{Au}$ $=$ autocoria e $\mathrm{Z}=$ zoocoria.

Table 1 - Structural parameters of tree species of an Atlantic Rainforest in Morro Grande, Santa Catarina State, Brazil, for individuals with $D B H \geq 5 \mathrm{~cm}$. Where: $H=$ maximum height $(\mathrm{m}) ; \mathrm{FA}=$ absolute frequency $(\%) ; D o A=$ absolute dominance $\left(\mathrm{m}^{2} . h \mathrm{a}^{-1}\right) ; \mathrm{DA}=$ absolute density (individuals. $\left.h \mathrm{a}^{-1}\right) ; \mathrm{VI}=$ importance value; Ec = ecological group; where $P$ = pioneer; $S i$ = initial secondary; $S t=$ late secondary, $C=$ climax; $P=$ pollination syndrome, where $A=$ anemophilic $; Z=$ zoophilic and $D=$ dispersal syndrome, where $A n=$ anemochoric; $A u=$ autochoric; and $Z=$ zoochoric.

\begin{tabular}{|c|c|c|c|c|c|c|c|c|}
\hline Espécie & $\mathrm{H}$ & FA & DoA & DA & VI & Ec & $\mathrm{P}$ & $\mathrm{D}$ \\
\hline Euterpe edulis Mart. & 14 & 72,4 & 9,815 & 645 & 23,7 & $\mathrm{C}$ & $\mathrm{Z}$ & $\mathrm{Z}$ \\
\hline Ficus adathodifolia Schott ex Spreng. & 20 & 8,0 & 12,213 & 43 & 8,4 & St & $\mathrm{Z}$ & Z \\
\hline Nectandra membranacea (Sw.) Griseb. & 19 & 9,6 & 5,134 & 55 & 4,8 & St & $\mathrm{Z}$ & $\mathrm{Z}$ \\
\hline Bathysa australis (A.St.-Hil.) K.Schum. & 12 & 19,2 & 1,434 & 114 & 4,7 & St & Z & An \\
\hline Hyeronima alchorneoides Allemão & 20 & 8,0 & 4,551 & 43 & 4,1 & $\mathrm{Si}$ & $\mathrm{Z}$ & $\mathrm{Z}$ \\
\hline Psychotria suterella Müll.Arg. & 7 & 16,8 & 0,577 & 106 & 3,8 & St & $\mathrm{Z}$ & Z \\
\hline Alchornea triplinervia (Spreng.) Müll.Arg. & 17 & 7,6 & 2,690 & 41 & 3,0 & $\mathrm{Si}$ & $\mathrm{Z}$ & Z \\
\hline Cabralea canjerana (Vell.) Mart. & 18 & 11,2 & 1,256 & 65 & 3,0 & St & $\mathrm{Z}$ & $\mathrm{Z}$ \\
\hline Alsophila setosa Kaulf. & 8 & 8,4 & 0,995 & 77 & 2,7 & $\mathrm{C}$ & - & An \\
\hline Matayba guianensis Aubl. & 15 & 7,6 & 1,912 & 47 & 2,6 & St & $\mathrm{Z}$ & $\mathrm{Au}$ \\
\hline Trichilia lepidota Mart. & 12 & 8,0 & 0,686 & 51 & 2,1 & $\mathrm{C}$ & Z & $\mathrm{Au}$ \\
\hline Gymnanthes concolor (Spreng.) Müll. Arg. & 10 & 8,8 & 0,476 & 49 & 2,0 & St & A & $\mathrm{Au}$ \\
\hline Casearia sylvestris $\mathrm{Sw}$. & 12 & 6,8 & 0,990 & 37 & 1,9 & $\mathrm{Si}$ & $\mathrm{Z}$ & $\mathrm{Z}$ \\
\hline Tetrorchidium rubrivenium Poepp. & 16 & 4,4 & 1,301 & 22 & 1,6 & $\mathrm{Si}$ & A & $\mathrm{Z}$ \\
\hline Allophylus edulis (A.St.-Hil. et al.) Hieron. ex Niederl. & 13 & 5,2 & 0,825 & 28 & 1,5 & $\mathrm{Si}$ & $\mathrm{Z}$ & Z \\
\hline Mollinedia schottiana (Spreng.) Perkins & 6 & 7,2 & 0,133 & 37 & 1,4 & $\mathrm{C}$ & $\mathrm{Z}$ & Z \\
\hline Cupania vernalis Cambess. & 17 & 4,0 & 0,709 & 22 & 1,2 & Pio & $\mathrm{Z}$ & Z \\
\hline Magnolia ovata (A.St.-Hi1.) Spreng. & 17 & 4,4 & 0,580 & 22 & 1,2 & St & Z & $\mathrm{Z}$ \\
\hline Cordia silvestris Fresen. & 15 & 2,4 & 1,147 & 12 & 1,1 & Pio & $\mathrm{Z}$ & An \\
\hline Endlicheria paniculata (Spreng.) J.F.Macbr. & 8 & 4,8 & 0,275 & 26 & 1,1 & St & $\mathrm{Z}$ & $\mathrm{Z}$ \\
\hline Rudgea jasminoides (Cham.) Müll.Arg. & 6 & 4,8 & 0,210 & 26 & 1,1 & $\mathrm{C}$ & $\mathrm{Z}$ & $\mathrm{Z}$ \\
\hline Pisonia zapallo Griseb. & 14 & 2,0 & 0,873 & 12 & 0,9 & $\mathrm{Si}$ & $\mathrm{Z}$ & Z \\
\hline Verbenoxylum reitzii (Moldenke) Tronc. & 13 & 3,6 & 0,352 & 18 & 0,9 & $\mathrm{Si}$ & Z & Z \\
\hline Luehea divaricata Mart. \& Zucc. & 14 & 2,0 & 0,861 & 10 & 0,9 & Pio & $\mathrm{Z}$ & Z \\
\hline Myrsine umbellata Mart. & 11 & 3,6 & 0,246 & 18 & 0,8 & $\mathrm{Si}$ & A & Z \\
\hline Cedrela fissilis Vell. & 15 & 2,4 & 0,585 & 12 & 0,8 & St & $\mathrm{Z}$ & Z \\
\hline Citronella paniculata (Mart.) R.A.Howard & 18 & 3,2 & 0,198 & 18 & 0,8 & $\mathrm{C}$ & $\mathrm{Z}$ & Z \\
\hline Citharexylum myrianthum Cham. & 15 & 1,2 & 0,819 & 8 & 0,7 & $\mathrm{Si}$ & $\mathrm{Z}$ & Z \\
\hline Nectandra oppositifolia Ness & 13 & 2,0 & 0,579 & 10 & 0,7 & St & $\mathrm{Z}$ & Z \\
\hline Myrcia tijucensis Kiaersk. & 10 & 2,8 & 0,170 & 14 & 0,6 & St & Z & $\mathrm{Z}$ \\
\hline Citrus $X$ limon (L.) Osbeck* & 7 & 2,8 & 0,067 & 16 & 0,6 & - & - & - \\
\hline Myrcia splendens (Sw.) DC. & 12 & 2,4 & 0,222 & 12 & 0,6 & $\mathrm{Si}$ & $\mathrm{Z}$ & $\mathrm{Z}$ \\
\hline Chrysophyllum inornatum Mart. & 9 & 2,0 & 0,260 & 10 & 0,5 & $\mathrm{C}$ & $\mathrm{Z}$ & $\mathrm{Z}$ \\
\hline Hovenia dulcis Thunb.* & 16 & 1,2 & 0,522 & 6 & 0,5 & - & - & - \\
\hline Annona rugulosa (Schltdl.) H.Rainer & 9 & 2,4 & 0,082 & 12 & 0,5 & St & $\mathrm{Z}$ & Z \\
\hline Calyptranthes grandifolia O. Berg & 8 & 2,4 & 0,049 & 12 & 0,5 & St & $\mathrm{Z}$ & Z \\
\hline Lamanonia ternata Vell. & 16 & 2,0 & 0,179 & 10 & 0,5 & Pio & Z & An \\
\hline Aspidosperma olivaceum Müll.Arg. & 7 & 2,4 & 0,041 & 12 & 0,5 & St & Z & An \\
\hline Sapium glandulosum (L.) Morong & 15 & 1,6 & 0,240 & 8 & 0,4 & Pio & $\mathrm{Z}$ & $\mathrm{Au}$ \\
\hline Nectandra megapotamica (Spreng.) Mez & 11 & 1,2 & 0,352 & 6 & 0,4 & St & $\mathrm{Z}$ & $\mathrm{Z}$ \\
\hline Virola bicuhyba (Schott ex Spreng.) Warb. & 11 & 2,0 & 0,080 & 10 & 0,4 & St & $\mathrm{Z}$ & $\mathrm{Z}$ \\
\hline
\end{tabular}

Revista Árvore, Viçosa-MG, v.39, n.1, p.49-58, 2015 
Tabela 1 - Cont...

Table 1 - Cont..

\begin{tabular}{|c|c|c|c|c|c|c|c|c|}
\hline Espécie & $\mathrm{H}$ & FA & DoA & DA & VI & Ec & $\mathrm{P}$ & $\mathrm{D}$ \\
\hline $\begin{array}{l}\text { Machaerium stipitatum Vogel } \\
\text { Myrsine hermogenesii (Jung-Mend. \& Bernacci) }\end{array}$ & 10 & 1,6 & 0,114 & 10 & 0,4 & Pio & Z & An \\
\hline M.F.Freitas \& Kin.-Gouv. & 9 & 2,0 & 0,048 & 10 & 0,4 & $\mathrm{Si}$ & A & $\mathrm{Z}$ \\
\hline Alchornea glandulosa Popp. \& Endl. & 13 & 0,8 & 0,398 & 4 & 0,4 & Pio & Z & Z \\
\hline Annona cacans Warm. & 16 & 0,8 & 0,392 & 4 & 0,4 & Pio & Z & Z \\
\hline Meliosma sellowii Urb. & 12 & 1,6 & 0,118 & 8 & 0,4 & St & Z & Z \\
\hline Posoqueria latifolia (Rudge) Schult. & 10 & 1,6 & 0,111 & 8 & 0,4 & $\mathrm{C}$ & Z & $\mathrm{Z}$ \\
\hline Guapira opposita (Vell.) Reitz & 8 & 1,6 & 0,087 & 8 & 0,4 & $\mathrm{Si}$ & Z & $\mathrm{Z}$ \\
\hline Erythrina falcata Benth. & 16 & 0,4 & 0,446 & 2 & 0,3 & $\mathrm{C}$ & Z & $\mathrm{Au}$ \\
\hline Guarea macrophylla Vahl & 9 & 1,2 & 0,155 & 6 & 0,3 & $\mathrm{C}$ & Z & $\mathrm{Z}$ \\
\hline Solanum sanctae-catharinae Dunal & 13 & 1,2 & 0,124 & 6 & 0,3 & Pio & Z & Z \\
\hline Cecropia glaziovii Snethl. & 19 & 0,8 & 0,218 & 4 & 0,3 & Pio & Z & $\mathrm{Z}$ \\
\hline Cyathea delgadii Sternb. & 3 & 1,2 & 0,061 & 6 & 0,3 & $\mathrm{C}$ & - & An \\
\hline Annona sericea Dunal & 9 & 1,2 & 0,029 & 6 & 0,2 & St & Z & $\mathrm{Z}$ \\
\hline Inga marginata Willd. & 8 & 1,2 & 0,033 & 6 & 0,2 & $\mathrm{Si}$ & Z & $\mathrm{Z}$ \\
\hline Dicksonia sellowiana Hook. & 3 & 0,8 & 0,137 & 4 & 0,2 & $\mathrm{C}$ & - & An \\
\hline Duguetia lanceolata A.St.-Hil. & 18 & 0,8 & 0,149 & 4 & 0,2 & St & Z & $\mathrm{Z}$ \\
\hline Roupala montana Aubl. & 12 & 0,8 & 0,142 & 4 & 0,2 & St & Z & An \\
\hline Ficus luschnathiana (Miq.) Miq. & 6 & 0,8 & 0,119 & 4 & 0,2 & St & $\mathrm{Z}$ & $\mathrm{Z}$ \\
\hline Campomanesia xanthocarpa (Mart.) O. Berg & 8 & 0,8 & 0,044 & 6 & 0,2 & $\mathrm{Si}$ & Z & $\mathrm{Z}$ \\
\hline Myrocarpus frondosus Allemão & 15 & 0,8 & 0,114 & 4 & 0,2 & $\mathrm{Si}$ & Z & An \\
\hline Myrcia brasiliensis Kiaersk. & 12 & 0,8 & 0,088 & 4 & 0,2 & St & Z & $\mathrm{Z}$ \\
\hline Cinnamomum glaziovii (Mez) Kosterm. & 20 & 0,4 & 0,193 & 2 & 0,2 & $\mathrm{C}$ & Z & $\mathrm{Z}$ \\
\hline Jacaranda puberula Cham. & 10 & 0,8 & 0,061 & 4 & 0,2 & Pio & Z & An \\
\hline Myrciaria plinioides D. Legrand & 10 & 0,8 & 0,047 & 4 & 0,2 & St & Z & $\mathrm{Z}$ \\
\hline Xylosma pseudosalzmanii Sleumer & 12 & 0,8 & 0,050 & 4 & 0,2 & $\mathrm{Si}$ & Z & $\mathrm{Z}$ \\
\hline Aegiphila integrifolia (Jacq.) Moldenke & 6 & 0,8 & 0,037 & 4 & 0,2 & Pio & Z & $\mathrm{Z}$ \\
\hline Myrcia pubipetala Miq. & 9 & 0,8 & 0,032 & 4 & 0,2 & St & $\mathrm{Z}$ & $\mathrm{Z}$ \\
\hline Sorocea bonplandii (Baill.) W.C. Burger et al. & 8 & 0,8 & 0,028 & 4 & 0,2 & St & $\mathrm{Z}$ & $\mathrm{Z}$ \\
\hline Trichilia pallens C.DC. & 13 & 0,8 & 0,027 & 4 & 0,2 & $\mathrm{C}$ & $\mathrm{Z}$ & $\mathrm{Au}$ \\
\hline Kaunia rufescens (Lund ex DC.) R.M. King \& H. Rob. & 6 & 0,8 & 0,019 & 4 & 0,2 & Pio & $\mathrm{Z}$ & An \\
\hline Maytenus sp. & 9 & 0,8 & 0,022 & 4 & 0,2 & St & Z & $\mathrm{Z}$ \\
\hline Ocotea indecora (Schott) Mez & 8 & 0,8 & 0,021 & 4 & 0,2 & $\mathrm{C}$ & Z & $\mathrm{Z}$ \\
\hline Xylopia brasiliensis Spreng. & 8 & 0,8 & 0,012 & 4 & 0,2 & St & Z & $\mathrm{Z}$ \\
\hline Zanthoxylum rhoifolium Lam. & 8 & 0,8 & 0,015 & 4 & 0,2 & $\mathrm{Si}$ & $\mathrm{Z}$ & $\mathrm{Au}$ \\
\hline Phytolacca dioica $\mathrm{L}$. & 9 & 0,4 & 0,074 & 2 & 0,1 & Pio & $\mathrm{Z}$ & $\mathrm{Z}$ \\
\hline Abarema langsdorffii (Benth.) Barneby \& J.W. Grimes & 13 & 0,4 & 0,048 & 2 & 0,1 & $\mathrm{Si}$ & Z & $\mathrm{Au}$ \\
\hline Myrciaria floribunda (H. West ex Willd.) O. Berg & 7 & 0,4 & 0,040 & 2 & 0,1 & $\mathrm{C}$ & $\mathrm{Z}$ & $\mathrm{Z}$ \\
\hline Pseudobombax grandiflorum (Cav.) A. Robyns & 17 & 0,4 & 0,038 & 2 & 0,1 & $\mathrm{Si}$ & Z & $\mathrm{Z}$ \\
\hline Rubiaceae 1 & 8 & 0,4 & 0,035 & 2 & 0,1 & St & Z & $\mathrm{Z}$ \\
\hline Schefflera angustissima (Marchal) Frodin & 9 & 0,4 & 0,047 & 2 & 0,1 & St & Z & $\mathrm{Z}$ \\
\hline Aegiphila brachiata Vell. & 6 & 0,4 & 0,023 & 2 & 0,1 & St & Z & $\mathrm{Z}$ \\
\hline Hirtella hebeclada Moric. Ex DC. & 7 & 0,4 & 0,028 & 2 & 0,1 & St & $\mathrm{Z}$ & $\mathrm{Z}$ \\
\hline Ilex theezans Mart. ex Reissek & 9 & 0,4 & 0,022 & 2 & 0,1 & Pio & Z & $\mathrm{Z}$ \\
\hline Marlierea silvatica (O. Berg) Kiaersk. & 7 & 0,4 & 0,020 & 2 & 0,1 & St & $\mathrm{Z}$ & $\mathrm{Z}$ \\
\hline Maytenus glaucescens Reissek & 16 & 0,4 & 0,020 & 2 & 0,1 & St & Z & $\mathrm{Z}$ \\
\hline Myrcia glabra (O. Berg) D. Legrand & 7 & 0,4 & 0,026 & 2 & 0,1 & St & $\mathrm{Z}$ & Z \\
\hline Prunus myrtifolia (L.) Urb. & 9 & 0,4 & 0,018 & 2 & 0,1 & $\mathrm{Si}$ & Z & $\mathrm{Z}$ \\
\hline Sloanea guianensis (Aubl.) Benth. & 8 & 0,4 & 0,018 & 2 & 0,1 & $\mathrm{C}$ & Z & $\mathrm{Z}$ \\
\hline Albizia edwallii (Hoehne) Barneby \& J.W. Grimes & 4 & 0,4 & 0,005 & 2 & 0,1 & St & Z & $\mathrm{Au}$ \\
\hline Annona neosericea H. Rainer & 5 & 0,4 & 0,011 & 2 & 0,1 & $\mathrm{Si}$ & Z & Z \\
\hline
\end{tabular}


Tabela 1 - Cont...

Table 1 - Cont...

\begin{tabular}{|c|c|c|c|c|c|c|c|c|}
\hline Espécie & $\mathrm{H}$ & FA & DoA & DA & VI & $\mathrm{Ec}$ & $\mathrm{P}$ & $\mathrm{D}$ \\
\hline Clethra scabra Pers. & 9 & 0,4 & 0,010 & 2 & 0,1 & Pio & $\mathrm{Z}$ & An \\
\hline Esenbeckia grandiflora Mart. & 7 & 0,4 & 0,005 & 2 & 0,1 & $\mathrm{Si}$ & Z & $\mathrm{Au}$ \\
\hline Eugenia bacopari D. Legrand & 4 & 0,4 & 0,004 & 2 & 0,1 & St & Z & $\mathrm{Z}$ \\
\hline Guatteria australis A.St.-Hil. & 7 & 0,4 & 0,017 & 2 & 0,1 & $\mathrm{St}$ & Z & Z \\
\hline Miconia саьиси Hoehne & 6 & 0,4 & 0,004 & 2 & 0,1 & Pio & $\mathrm{Z}$ & $\mathrm{Z}$ \\
\hline Mollinedia sp. & 5 & 0,4 & 0,004 & 2 & 0,1 & $\mathrm{C}$ & $\mathrm{Z}$ & Z \\
\hline Myrsine parvula (Mez) Otegui & 11 & 0,4 & 0,004 & 2 & 0,1 & $\mathrm{Si}$ & A & $\mathrm{Z}$ \\
\hline Ocotea catharinensis $\mathrm{Mez}$ & 5 & 0,4 & 0,006 & 2 & 0,1 & $\mathrm{C}$ & $\mathrm{Z}$ & $\mathrm{Z}$ \\
\hline Piptocarpha axillaris (Less.) Baker & 8 & 0,4 & 0,001 & 2 & 0,1 & Pio & $\mathrm{Z}$ & An \\
\hline Piptocarpha tomentosa Baker & 6 & 0,4 & 0,006 & 2 & 0,1 & Pio & $\mathrm{Z}$ & An \\
\hline Solanum pseudoquina A.St.-Hil. & 7 & 0,4 & 0,006 & 2 & 0,1 & Pio & $\mathrm{Z}$ & $\mathrm{Z}$ \\
\hline Xylosma prockia (Turcz.) Turcz. & 8 & 0,4 & 0,016 & 2 & 0,1 & $\mathrm{Si}$ & $\mathrm{Z}$ & $\mathrm{Z}$ \\
\hline Total & & 319 & 59 & 2.034 & 100 & & & \\
\hline
\end{tabular}

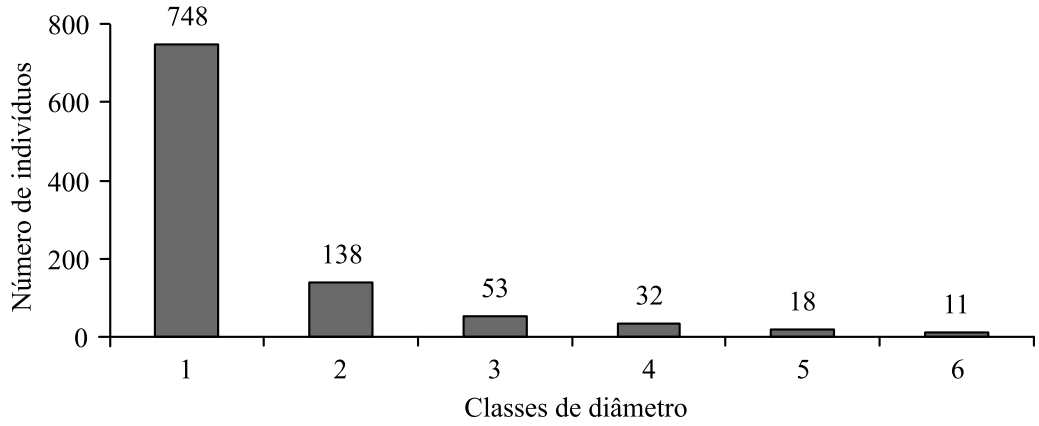

Figura 1 - Distribuição do número de indivíduos arbóreos por classe de diâmetro, na Floresta Ombrófila Densa Montana, no Município de Morro Grande, Santa Catarina, em que 1 =5-14,9 cm; $2=15-24,9 \mathrm{~cm} ; 3=25-34,9 \mathrm{~cm} ; 4$ $=35-44,9 \mathrm{~cm} ; 5=45-54,9 \mathrm{~cm} ;$ e $6=\geq 55 \mathrm{~cm}$.

Figure 1 - Distribution of the number of trees in diameter classes of an Atlantic Rainforest in Morro Grande, Santa Catarina State, Brazil, where: $1=5-14.9 \mathrm{~cm} ; 2=15-24.9 \mathrm{~cm} ; 3=25-34.9 \mathrm{~cm} ; 4=35-44.9 \mathrm{~cm} ; 5=45-54.9 \mathrm{~cm}$; and $6=\geq 55 \mathrm{~cm}$.

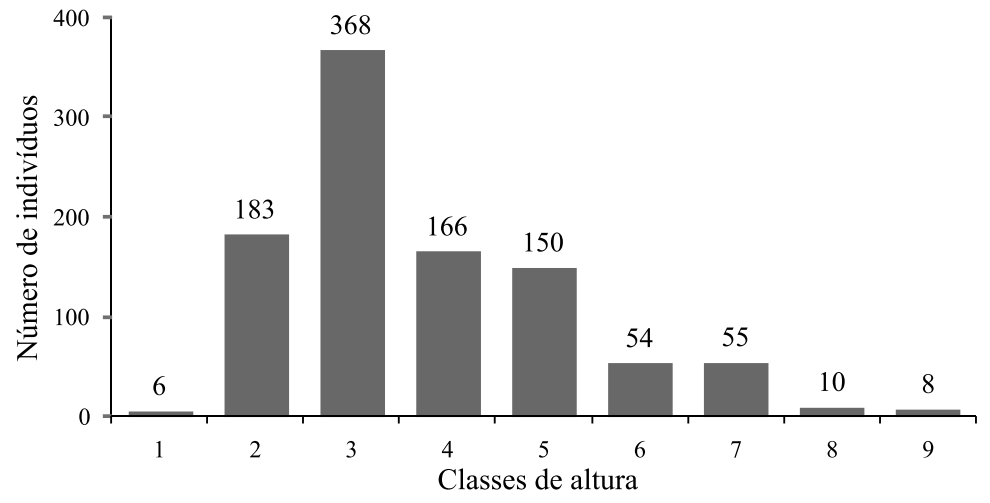

Figura 2 - Distribuição do número de indivíduos arbóreos por classe de altura, na Floresta Ombrófila Densa Montana, no Município de Morro Grande, Santa Catarina, sendo $1=2-2,4 \mathrm{~m} ; 2=2,5-4,9 \mathrm{~m} ; 3=5-7,4 \mathrm{~m} ; 4$ = 7,5-9,9 m; $5=10-12,4 \mathrm{~m} ; 6=12,5-14,9 \mathrm{~m} ; 7=15-17,4 \mathrm{~m} ; 8=17,5-19,9 \mathrm{~m} ;$ e $9=\geq 20 \mathrm{~m}$.

Figure 2 - Distribution of number of trees in height classes of an Atlantic rain forest in Santa Catarina State, Brazil. Where: $1=2-2,4 \mathrm{~m} ; 2=2,5-4,9 \mathrm{~m} ; 3=5-7,4 \mathrm{~m} ; 4=7,5-9,9 \mathrm{~m} ; 5=10-12,4 \mathrm{~m} ; 6=12,5-14,9 \mathrm{~m} ; 7=15-17,4 \mathrm{~m}$; $8=17,5-19,9 m ;$ and $9=\geq 20$.

Revista Árvore, Viçosa-MG, v.39, n.1, p.49-58, 2015 
$95 \%$ apresentaram polinização zoofílica e 77\%, dispersão zoocórica (Tabela 1).

\section{DISCUSSÃO}

Na Região Sul do Brasil, Myrtaceae é a família dominante em diversos estudos (CITADINI-ZANETTE et al., 2003; SANTOS; LEAL-FILHO, 2003; COLONETTI et al., 2009; KLAUBER et al., 2010; SANTOS et al., 2012, entre outros).

No local de estudo, E. edulis desenvolveu-se no sub-bosque, não demonstrando preferência quanto à topografia da área e às condições locais do solo (declividade, profundidade, umidade), comprovado pelo valor de frequência obtido (72\%). Diversos estudos da estrutura da comunidade arbórea apontaram E. edulis com maior valor de importância, principalmente pelos altos valores de densidade, nas formações florestais (BORÉM; OLIVEIRA-FILHO, 2002; CAMPOS et al., 2006), o que deve estar relacionado ao seu grande sucesso reprodutivo e à grande disponibilidade de frutos produzidos anualmente (REIS; KAGEYAMA, 2000). E. edulis sofre frequentes cortes seletivos e clandestinos, pois é abundante em toda a Floresta Ombrófila Densa.

A frequência de classes de altura e de diâmetro, da população de E. edulis, representou a espécie de maior destaque na área de estudo. A distribuição dos indivíduos de E. edulis por classe de altura pode ser reflexo das condições da área de estudo, utilizada para lavoura e com abate dos indivíduos adultos e num passado recente (ocorrido há aproximadamente 30 anos), para retirada de lenha, fato que deve ter levado a uma recomposição natural mais lenta pela diminuição das plantas matrizes.

A distribuição por classe de diâmetro mostrou que a maior concentração de indivíduos das espécies arbóreas está na terceira, com diâmetros entre $10 \mathrm{~cm}$ e $12,5 \mathrm{~cm}$, comportamento esse também observado em florestas no Rio Grande do Sul (JARENKOW, 1994) e em Santa Catarina (CITADINI-ZANETTE, 1995).

Nos ecossistemas, as interações biológicas, são resultado da evolução que acabaram moldando as adaptações de certas espécies à presença de outras, servindo de subsídio para determinada espécie se manter presente nesse ambiente (JOLY et al., 2011). Embora os estudos avancem na direção de outras interações interespecíficas, a polinização e a dispersão de propágulos constituem ainda a maior contribuição para o retorno da fauna em ambientes alterados. A fauna associada à vegetação é considerada componente-chave para a manutenção do processo dinâmico-sucessional nas formações florestais secundárias, destacando-se as interações que ocorrem na polinização e dispersão (ZOUCAS et al., 2004).

O aumento da fragmentação florestal e de áreas degradadas diminui as populações de muitas espécies de plantas nativas e seus polinizadores. Há previsão de que muitas espécies de plantas dentro das próximas décadas apresentarão declínios em suas populações devido à relação de interdependência com seus polinizadores (ALLEN-WARDELL et al., 1998). Além da polinização, a dispersão é outra importante interação entre fauna e vegetação. A dispersão de sementes constitui mecanismo essencial para a dinâmica da floresta, influenciando, consequentemente, a regeneração natural das populações (ZAMBONIM, 2001; TABARELLI; PERES, 2002).

Neste estudo, do total de espécies analisadas, a dispersão zoocórica foi característica da maioria das espécies. Na dispersão, os termos espécie-chave, keystone (SMYTHE, 1986) e bagueira, usados pelos caçadores de Santa Catarina (REIS et al., 1999), podem ser considerados sinônimos e definem espécies que apresentam alimentos disponíveis o ano todo. Muitas Arecaceae, Moraceae e Myrtaceae (p. ex. Euterpe edulis, Ficus adhatodifolia e Myrcia splendens) são consideradas bagueiras e podem representar estratégia básica para atrair rapidamente o maior número de animais, aumentando a resiliência do ambiente.

Parte da estrutura de uma floresta pode ser avaliada por sua distribuição diamétrica, a qual é definida pela caracterização do número de árvores por unidade de área e por intervalos de classes de diâmetros (PIRES et al., 1995). Dos indivíduos amostrados, 75\% se encontraram com valores de diâmetro menores de $15 \mathrm{~cm}$. O elevado número de indivíduos em classe menores de diâmetro pode ser atribuído ao critério de inclusão adotado e ao fato de a comunidade estar representada por indivíduos de pequeno porte, devido à intensa dinâmica de grande parte dessa área, que anos atrás servia para agricultura e passou por processo seletivo de exploração madeireira.

Neste estudo, as categorias mais tardias (secundárias tardias e clímax) perfizeram aproximadamente 59\% do

Revista Árvore, Viçosa-MG, v.39, n.1, p.49-58, 2015 
total do número de indivíduos amostrados, evidenciando que a floresta se direciona para fases mais avançadas de sucessão ecológica secundária.

As espécies de início da sucessão desempenham alto valor ecológico na comunidade durante o processo sucessional, pelo fato de se desenvolverem em clareiras e em áreas degradadas, apresentarem rápido crescimento, ciclo de vida curto, produzir muitas sementes dispersas por agentes generalistas e formarem banco de sementes com viabilidade por longo período (WHITMORE, 1978; GÓMEZ-POMPA; VASQUEZ-YANES, 1981). Rodrigues (1995) comentou que as espécies pioneiras têm função cicatrizadora de ambientes perturbados, favorecidas pela maior luminosidade e condicionadas à presença e viabilidade de diásporos, sendo estas a principal fonte de mudanças estruturais. No outro extremo das pioneiras, têm-se as clímax, que são as espécies finais na substituição sequencial da sucessão. Entre os dois extremos, existe grande número de espécies com características ou adaptações ecológicas intermediárias. Quando as características se aproximaram mais com as das pioneiras, essas espécies são chamadas de secundárias iniciais; quando apresentam características mais próximas das espécies clímax, são denominadas secundárias tardias.

Foram amostradas duas espécies exóticas, introduzidas por antigos moradores que habitaram as proximidades da área estudada. Citrus x limon (L.) Osbeck e Hovenia dulcis Thunb. (originárias da Ásia) constam na lista oficial das espécies exóticas invasoras no Estado de Santa Catarina, cujo manejo e cultivo são permitidos sob condições controladas (CONSEMA, 2012). Holvenia dulcis é altamente agressiva e apontada como uma das mais importantes espécies na invasão biológica de ecossistemas no Sul do Brasil.

\section{CONCLUSÃO}

A Mata Atlântica, que abriga parcela significativa da diversidade biológica do Brasil, vem sofrendo com ações expressivas de perdas de hábitat de muitas espécies endêmicas. Tendo em vista a escassez de informações sobre Floresta Ombrófila Densa Montana, há necessidade de estudos para melhor compreensão dessa formação no Estado de Santa Catarina. A floresta foi classificada como de estágio avançado de regeneração natural, com base em sua composição florística e estrutural, após 30 anos da última intervenção humana na área.

Revista Árvore, Viçosa-MG, v.39, n.1, p.49-58, 2015
A interação entre vegetação e fauna evidenciou a importância dessa relação para ações de manejo e recuperação de áreas alteradas na região. Zoofilia e zoocoria mostraram-se as mais importantes síndromes de polinização e de dispersão neste estudo, eventos característicos nas formações da Floresta Ombrófila Densa, principalmente daquelas em estágios sucessionais avançados de regeneração natural. O conhecimento produzido na área estudada poderá, no futuro, fornecer alternativas de uso e manejo de outras áreas similares, sendo sua manutenção importante para estimular a preservação de outros remanescentes florestais na região. As informações obtidas no levantamento, além de contribuir para o conhecimento da biodiversidade regional, poderão servir para a restauração ecológica de remanescentes florestais alterados, com indicação das interações interespecíficas na manutenção e preservação das florestas.

\section{REFERÊNCIAS}

ALLEN-WARDELL, G.; BERNHARDT, P.; BITNER, $\mathrm{R}$. The potential consequences of pollinator declines on the conservation of biodiversity and stability of food crop yields. Conservation Biology, v.12, n.1, p.8-17, 1998.

APG. Angiosperm Phylogeny Group. An update of the Angiosperm Phylogeny Group classification for the orders and families of flowering plants: APG III. Botanical Journal of the Linnean Society, n.161, p.105-121, 2009.

ARRUDA, M. B. (Org.). Ecossistemas brasileiros. Brasília: IBAMA, 2001. 49p.

BORÉM, R. A. T.; OLIVEIRA-FILHO, A. T. Fitossociologia do estrato arbóreo em uma toposequência alternada de mata atlântica, no munícipio de Silva Jardim, RJ, Brasil. Revista Árvore, v.26, n.6, p.727-742, 2002.

BRITO, F. Corredores ecológicos: uma estratégia integradora na gestão de ecossistemas. Florianópolis: UFSC, 2006.

CAMPOS, E. P.; SILVA, A. F.; MEIRA NETO, J. A. A.; MARTINS, S. V. Florística e estrutura horizontal da vegetação arbórea de uma ravina em um fragmento florestal no município de Viçosa, MG. Revista Árvore, v.30, n.6, p.1045-1054, 2006. 
CITADINI-ZANETTE, V. Florística, fitossociologia e aspectos da dinâmica de um remanescente de Mata Atlântica na microbacia do rio Novo, Orleans, SC. 1995. 236f. Tese (Doutorado em Ecologia) - Universidade Federal de São Carlos, São Carlos, 1995.

CITADINI-ZANETTE, V.; SANTOS, R.; REMUS, G. SOBRAL, M. Myrtaceae do Sul de Santa Catarina: subsídio para recuperação de ecossistemas degradados. Revista deTecnologia e Ambiente, v.9, n.2, p.6175, 2003.

COLONETTI, S.; CITADINI-ZANETTE, V.; MARTINS, R.; SANTOS, R.; ROCHA, E.; JARENKOW, J. A. Florística e estrutura fitossociológica em Floresta Ombrófila Densa Submontana na barragem do rio São Bento, Siderópolis, Estado de Santa Catarina. Acta Scientiarum Biological Sciences, v.31, n.4, p.397-405, 2009.

CONSEMA. Resolução n. 08, de 14 de setembro de 2012. Reconhece a lista oficial de espécies exóticas invasoras de Santa Catarina. Diário Oficial da União, n. 19429, 2 out. 2012.

COTTAM, G.; CURTIS, J. T. The use of distance measures in phiytosociological sampling. Ecology, v.37, p.451-460, 1956.

FAEGRI, K.; van der PIJL, L. The principles of pollination ecology. Oxford: Pergamon Press, 1979.

FERRETTI, A. R.; KAGEYAMA, P. Y.; ÁRBOEZ, G. F.; SANTOS, J. D.; BARROS, M. I. A.; LORZA, R. F.; OLIVEIRA, C. Classificação das espécies arbóreas em grupos ecológicos para revegetação com nativas no Estado de São Paulo. Florestar Estatístico, v.3, n.7, p.7377, 1995.

GÓMEZ-POMPA, A. G.; VÁZQUEZ-YANES, C. N. Successional studies of a rain forest in Mexico, In: WEST, D. C.; SCHUGART, H. H.; BOTKIN, D. B. (Ed.). Forest concepts and application. New York: Springer-Verlag, 1981. p.247-266.

JARENKOW, J. A. Estudos fitossociológicos comparativos entre duas áreas com mata de encosta no Rio Grande do
Sul. 1994. 122f. Tese (Doutorado em Ecologia e Recursos Naturais) - Universidade Federal de São Carlos, São Carlos, 1994.

JOLY, A. C.; HADDAD, C. F. B.; VERDADE, L. M.; OLIVEIRA, M. C.; BOLZANI, V. S.; BERLINCK, R. G. S. Diagnóstico da pesquisa em biodiversidade no Brasil. Revista USP, n. 89, p.114-133, 2011.

LISTA DE ESPÉCIES DA FLORA DO BRASIL. Jardim Botânico do Rio de Janeiro. Disponível em: <http://floradobrasil.jbrj.gov.br/>. Acesso em: 18 ago. 2014.

MUELLER-DOMBOIS, D.; ELLENBERG, H. Aims and methods of vegetation ecology. New York: Wiley, 1974.

MYERS, N.; MITTERMEIER, R. A.; MITTERMEIER, C. G.; FONSECA, G. A. B.; KENT, J. Biodiversity hotspots for conservation priorities. Nature, v.403, p.853-845, 2000.

NASCIMENTO, D. Agricultura e mineração na formação do espaço urbano de Criciúma.

Revista Ciências Humanas, v.9, n.1, p.7$16,2003$.

PANDOLFO, C.; BRAGA, H. J.; SILVA JÚNIOR, V. P.; MASSIGNAN, A. M.; PEREIRA, E. S.; THOMÉ, V. M. R.; VALCI, F. V. Atlas climatológico do Estado de Santa Catarina. Florianópolis: Epagri, 2002. CD-ROM

PIRES-O'BRIEN, M. J.; O'BRIEN, C. M. Ecologia e modelamento de florestas tropicais. Belém: FCAP/Serviço de Documentação e Informação, 1995.

REIS, A.; KAGEYAMA, P. Y. Dispersão de sementes do palmiteiro (Euterpe edulis MartiusPalmae). In: REIS, A.; REIS, M. S. (Ed.). Euterpe edulis Martius - palmiteiro: biologia, conservação e manejo. Itajaí: Herbário Barbosa Rodrigues, 2000. p.60-92.

REIS, A.; ZAMBONIN, R. M.; NAKAZONO, E. $M$. Recuperação de áreas florestais degradadas utilizando a sucessão e as interações planta-animal. São Paulo: Conselho Nacional da Reserva da Biosfera da Mata Atlântica/UNESCO, 1999.

Revista Árvore, Viçosa-MG, v.39, n.1, p.49-58, 2015 
RIBEIRO, M. C.; METZGER, J. P.; MARTENSEN, A. C.; PONZONI, F. J.; HIROTA, M. M. The Brazilian Atlantic Forest: How much is left, and how is the remaining forest distributed? Implications for conservation. Biological Conservation, v.142, p.1141-1153, 2009.

RODRIGUES, R. R. A sucessão florestal. In: MORELlATO, P. C.; LEITÃO-FILHO, H. F. (Org.). Ecologia e preservação de uma floresta tropical urbana: Reserva Santa Genebra, 1995. p. 30-36.

SANTOS, R.; LEAL-FILHO, L. S. Reabilitação de ecossistemas degradados pela mineração de carvão a céu aberto em Santa Catarina, Brasil. São Paulo: Escola Politécnica da USP, BT/PMI/205, 2003. 20p. (Boletim Técnico)

SANTOS, R.; SILVA, R. C.; PACHECO, D.; MARTINS, R.; CITADINI-ZANETTE, V. Florística e estrutura do componente arbustivo-arbóreo de mata de restinga arenosa no Parque Estadual de Itapeva, Rio Grande do Sul. Revista Árvore, v.36, n.6, p.1047-1059, 2012.

SCHEIBE, L. F.; BUSS, M. D.; FURTADO, S. M. A. Atlas Ambiental da Bacia do rio Araranguá, Santa Catarina, Brasil. Florianópolis: UFSC/Cidade Futura, 2010.
SMYTHE, N. The importance of mamals in neotropical forest management. In: COLÓN, J. C. (Ed.). Management of the forest of tropical America: prospects and tecnologies. Puerto Rico: Departament of Agriculture Forest Service, 1986. p.79-98.

TABARELLI, M.; PERES, C. A. Abiotic and vertebrate seed dispersal in the Brazilian Atlantic Forest: implications for forest regeneration. Biological Conservation, v.106, p.165-176, 2002.

van der PIJL, L. Principles of dispersal in higher plants. 3.ed. New York: Springer-Verlag, 1982.

WHITMORE, T. C. Gaps in the forest canopy. In: TOMLINSON, Z. Tropical trees as living systems. London: Cambridge University Press, 1978.

ZAMBONIM, R. M. Banco de dados como subsídio para conservação e restauração nas tipologias vegetacionais do Parque Estadual da Serra do Tabuleiro. 2001. 118f. Dissertação (Mestrado em Recursos Genéticos Vegetais) - Universidade Federal de Santa Catarina, Florianópolis, 2001.

ZOUCAS, B. C.; CITADINI-ZANETTE, V.; SANTOS, R. Relações interespecíficas na recuperação de áreas degradadas. Revista Tecnologia e Ambiente, v.10, n.2, p.81-97, 2004. 